\title{
A longitudinal study of mental wellbeing in students that transition into PhD study.
}

Taylor Winter ${ }^{\mathrm{a}}$, Benjamin C. Riordan ${ }^{\mathrm{b}}$, John A. Hunter ${ }^{\mathrm{c}}$, Karen Tustin ${ }^{\mathrm{c}, \mathrm{d}}$, Megan Gollop ${ }^{\mathrm{e}}$, Nicola Taylor ${ }^{\mathrm{f}}$, Jesse Kokaua ${ }^{\mathrm{g}, \mathrm{h}}$, Richie Poulton ${ }^{\mathrm{c}, \mathrm{d}, \mathrm{h}}, \&$ Damian Scarf ${ }^{\mathrm{c}, \mathrm{i}^{*}}$

${ }^{a}$ Department of Psychology, Victoria University of Wellington, Wellington, New Zealand

${ }^{b}$ Discipline of Addiction Medicine, Central Clinical School, Faculty of Medicine and Health, University of Sydney, Sydney, Australia

${ }^{c}$ Department of Psychology, University of Otago, New Zealand

${ }^{d}$ National Centre for Lifecourse Research, University of Otago, Dunedin, New Zealand

${ }^{e}$ Children's Issues Centre, University of Otago, Dunedin, New Zealand

${ }^{f}$ Division of Health Sciences, University of Otago, Dunedin, New Zealand

${ }^{g}$ Pacific Development Office, University of Otago, Dunedin, New Zealand

${ }^{h}$ Dunedin Multidisciplinary Health and Development Research Unit, University of Otago, Dunedin, New Zealand

${ }^{i}$ Office of Student Success, University of Otago, Dunedin, New Zealand

*Damian Scarf, E-mail: damian@psy.otago.ac.nz

Department of Psychology, University of Otago, New Zealand. 


\title{
A longitudinal study of mental wellbeing in students that transition into PhD study.
}

\author{
Journal editorials, career features, and the popular press, commonly talk of a \\ graduate student mental health crisis. To date, studies on graduate student mental \\ health have employed cross-sectional designs, limiting any causal conclusions \\ regarding the relationship between graduate student and mental health. Here, we \\ present the first longitudinal study on mental health in $\mathrm{PhD}$ students. Data were \\ drawn from a longitudinal study of undergraduate students in New Zealand, \\ allowing us to compare students who did, and did not, transition into PhD study \\ following the completion of their undergraduate degree. Using multilevel \\ Bayesian regression, we detected a 0.09 standard deviation decrease in mental \\ health for students who enter $\mathrm{PhD}$ study. This finding is orders of magnitude \\ smaller than one might expect based on previous cross-sectional research and \\ provide an important message; that poor mental health is not an inevitable \\ consequence of graduate study.
}

Keywords: graduate student; mental health; longitudinal

\section{Introduction}

There is a plethora of editorials and career features on graduate student mental health in high impact journals, many with alarming titles such as "Caught in a trap" (Sohn 2016), "Paying graduate school's mental toll" (Arnold 2014), and "The tortuous truth" (Woolston 2019). The results from Nature's $2019 \mathrm{PhD}$ survey do little to quell the fears of future PhD students. Of the 6,300 early-career researchers surveyed across North America, South America, Africa, Asia, Australia, and Europe, 36\% had sought help for anxiety or depression (Woolston 2019).

In one of the few empirical studies on this topic, anxiety and depression were assessed in 2,279 graduate students (Evans et al. 2018). Although the students were drawn from 26 countries, $92 \%$ of the students were based at a US institution. Prevalence rates for anxiety and depression were alarmingly high, with $41 \%$ having moderate to severe 
anxiety and $32 \%$ having moderate to severe depression. These prevalence rates are six times higher than those of the general population, leading the authors to conclude that there is a mental health crisis in the graduate student population (Evans et al. 2018).

Rather than being unique to US institutions, research from Europe also provides a bleak picture for future $\mathrm{PhD}$ students. A survey of 3,659 $\mathrm{PhD}$ students in Belgium revealed that a high proportion of students $(32 \%)$ reported at least four symptoms of psychological distress (Levecque et al. 2017). For comparison purposes, the $\mathrm{PhD}$ student data were compared to a sample of highly educated individuals from the general population. Although lower than the relative prevalence rate reported for students based at US institutions, PhD students had a prevalence of having or developing a psychiatric disorder that was 2.43 times higher than the comparison sample (Levecque et al. 2017).

To date, studies on graduate student mental health have been crosssectional, limiting any discussion regarding the temporal link between graduate education and mental health. To address this limitation, we used data from the Graduate Longitudinal Study New Zealand (GLSNZ). The GLSNZ conducted baseline sampling across all eight New Zealand universities between July and December 2011 (Tustin et al. 2012) and followed up this sample in 2014 (Tustin et al. 2016).

For the current study, we focused on the 269 students who transitioned into PhD study between the baseline survey (2011) and the follow-up survey (2014). All participants completed the validated Warwick-Edinburgh Mental Well-being Scale (WEMWBS) (Tennant et al. 2007), basic demographic information, and a measure of economic strain. To determine whether any changes observed in $\mathrm{PhD}$ students were the direct result of the transition into graduate study, we compared $\mathrm{PhD}$ students with the 4,230 students who also graduated in 2011 but did not transition into further study. 


\section{Materials and Methods}

\section{Participants}

We collected data from 8,719 participants in 2011, of whom 5983 completed all questions used in our analysis, including the follow-up questions in 2014 (Tustin et al. 2012; Tustin et al. 2016). We then removed 367 participants who were $\mathrm{PhD}$ candidates in 2011 and removed 1117 participants who were in some other form of study in 2014, resulting in a final sample of 269 participants who transitioned into a $\mathrm{PhD}$ study in 2014 and 4230 participants who did not enter some form of study in 2014. Each wave of the study was reviewed and approved by the University of Otago Human Ethics Committee. Participants provided informed consent at the start of each survey.

\section{Measures}

\section{Demographic Information}

Participants provided demographic information at baseline, including age, sex, and ethnicity. Additionally, participants were separated into two groups, those who transitioned into PhD study between the 2011 and 2014 surveys (i.e., PhD entrants) and those who did not enter into $\mathrm{PhD}$ study after completing their qualification in 2011 (i.e., PhD non-entrants).

\section{Economic Strain}

The current financial situation of participants was collected using five questions and responses provided using a five-point Likert scale. Questions asked whether participants had enough money to afford items such as accommodation, or if they have had trouble meeting financial commitments. We summed responses for all five items, with a minimum score of five and maximum of 25 , with higher scores reflecting less economic 
strain.

\section{Mental Wellbeing}

We estimated mental wellbeing using the WEMWBS, which consists of 14 items asking about how participants felt over the last two weeks (Tennant et al. 2007). Responses were provided using five-point Likert scales which were summed to produce a score ranging from 14 to 70, where higher scores reflected better mental wellbeing.

Analysis

All continuous variables were standardised and centred on zero. We then analysed the data using multilevel Bayesian regression with the brms package in R (Bürkner 2017). We modelled mental wellbeing as a dependent variable with age, sex, economic strain, time, and $\mathrm{PhD}$ entrance as covariates. To test our hypothesis that wellbeing decreases for $\mathrm{PhD}$ entrants relative to $\mathrm{PhD}$ non-entrants, we included an interaction between time and $\mathrm{PhD}$ transition. We then used random intercepts for participants to account for repeated measures. Lastly, pairwise comparisons were conducted by removing the main effect of time and $\mathrm{PhD}$ entrance so that the interaction term in the model produced estimates for each combination of time (2011 vs 2014) and group (PhD entrant vs $\mathrm{PhD}$ non-entrant) separately.

Construction of priors: Our priors were based on three previous studies that suggested PhD students had 1.5 standard deviations lower mental wellbeing (Marais et al. 2018), $\mathrm{PhD}$ students were six times more likely to experience anxiety or depression (Evans et al. 2018), and $\mathrm{PhD}$ students were 2.43 times more likely to develop a psychological condition (Levecque et al. 2017). Taken together and considering some chance that effects are smaller in our New Zealand sample, we used an informed prior for the 
interaction between time and $\mathrm{PhD}$ entrance that took the form of a normal distribution with a standardised mean of -1 and a standard deviation of 0.4 . All other variables had a weakly-informative normal prior with a mean of zero and a standard deviation of 0.5 . In both cases, we intentionally adopted a standard deviation that would give reasonable chance of an effect being approximately zero, i.e., null.

\section{Results and Discussion}

Multilevel Bayesian regression yielded over a $99.9 \%$ posterior probability of an interaction between time and $\mathrm{PhD}$ transition (Table 1). Pairwise comparisons between the two time points for each group indicated that $\mathrm{PhD}$ non-entrants experienced an increase in their level of mental wellbeing in 2014 relative to their level of mental wellbeing in 2011 (posterior probability > 99\%), whereas $\mathrm{PhD}$ entrants presented with lower levels of mental wellbeing in 2014 relative to their levels of mental wellbeing in 2011 (posterior probability $=92 \%$ ). Thus, in 2011 , there was no difference between the two groups (posterior probability $=54 \%$ ) but, in 2014 , we saw that PhD entrants had significantly lower mental wellbeing than did those who did not transition to $\mathrm{PhD}$ studies, posterior probability $>99 \%$ (Figure 1).

The current study represents the first attempt to track mental wellbeing of students as they transition from completing their undergraduate degree into $\mathrm{PhD}$ study. $\mathrm{PhD}$ entrants displayed a decrease in mental wellbeing between 2011 and 2014. The decrease, however, was extremely small and orders of magnitude smaller than one might expect based on previous empirical work (Garcia-Williams et al. 2014; Levecque et al. 2017; Evans et al. 2018) and coverage in major journals (Arnold 2014; Sohn 2016; Woolston 2017, 2019). Moreover, there was no evidence that $\mathrm{PhD}$ entrants displayed lower levels of mental wellbeing at the baseline assessment, providing no evidence that those who enter a $\mathrm{PhD}$ are a self-selecting sample of people with lower mental wellbeing. 
An important question is why our findings differ from previous cross-sectional studies on $\mathrm{PhD}$ student mental health? One source of difference is the structure of New Zealand PhD programmes. Relative to North American programmes, New Zealand programmes have little (if any) course work. Although they are more similar to the European programmes, there are still a number of differences. For example, median timeto-degree in New Zealand is shorter ( 4 vs. $~ 5$ years) (Levecque et al. 2017; SpronkenSmith et al. 2018) and completion rates are higher (66\% vs. 80\%) (Spronken-Smith et al. 2018; Hasgall et al. 2019).

A second source of difference is that the current study employed a measure of mental wellbeing (i.e., the WEMWBS), while previous studies have tended to employ measures of depression, anxiety, or general psychological distress. For example, Levecque et al. (2017) employed the GHQ-12. While different on the surface, Böhnke and Croudace (2016) recently demonstrated that the GHQ-12 and WEMWBS share a single dimension, with the lack of differentiation suggesting that they provide comparable measures of general health and mental wellbeing. Perhaps one benefit of the WEMWBS over these other measures is that it takes a more positive approach to assessing mental health. Indeed, emerging adults are already viewed through a deficit lens (Trzesniewski and Donnellan 2014; Arahanga-Doyle et al. 2019), and reports on the prevalence of depression, anxiety, and general psychological distress of $\mathrm{PhD}$ students may contribute to this general depiction. At the very least, studies that assess mental health in $\mathrm{PhD}$ students should complement their analysis with a strengths-based or mental wellbeing approach.

Rather than representing limitations, the above differences may provide a pathway to improving the mental health of graduate students in other countries. Above all, the 
current study demonstrates that poor mental health is not an inevitable consequence of graduate study.

Acknowledgements: We thank the participants and participating universities who facilitated this study.

\section{References}

Arahanga-Doyle H, Moradi S, Brown K, Neha T, Hunter JA, Scarf D. 2019. Positive youth development in Māori and New Zealand European adolescents through an adventure education programme. Kōtuitui: New Zealand Journal of Social Sciences Online. 14(1):38-51.

Arnold C. 2014. Paying graduate school's mental toll. [accessed]. https://www.sciencemag.org/careers/2014/02/paying-graduate-schools-mental-toll. Böhnke JR, Croudace TJ. 2016. Calibrating well-being, quality of life and common mental disorder items: psychometric epidemiology in public mental health research. The British Journal of Psychiatry. 209(2):162-168.

Bürkner P-C. 2017. brms: An R package for Bayesian multilevel models using Stan. Journal of Statistical Software. 80(1):1-28.

Evans TM, Bira L, Gastelum JB, Weiss LT, Vanderford NL. 2018. Evidence for a mental health crisis in graduate education. Nat Biotechnol. 36(3):282-284.

Garcia-Williams AG, Moffitt L, Kaslow NJ. 2014. Mental health and suicidal behavior among graduate students. Academic Psychiatry. 38(5):554-560.

Hasgall A, Saenen B, Borrell-Damian L, Van Deynze F, Seeber M, Huisman J. 2019. Doctoral education in Europe today: approaches and institutional structures. Switzerland. 
Levecque K, Anseel F, De Beuckelaer A, Van der Heyden J, Gisle L. 2017. Work organization and mental health problems in PhD students. Research Policy. 46(4):868879.

Marais GA, Shankland R, Haag P, Fiault R, Juniper B. 2018. A survey and a positive psychology intervention on French PhD student wellbeing. International Journal of Doctoral Studies. 13:109-138.

Sohn E. 2016. Mental health: Caught in a trap. Nature. 539:319-321.

Spronken-Smith R, Cameron C, Quigg R. 2018. Factors contributing to high PhD completion rates: a case study in a research-intensive university in New Zealand. Assessment \& Evaluation in Higher Education. 43(1):94-109.

Tennant R, Hiller L, Fishwick R, Platt S, Joseph S, Weich S, Parkinson J, Secker J, Stewart-Brown S. 2007. The Warwick-Edinburgh mental well-being scale (WEMWBS): development and UK validation. Health and Quality of life Outcomes. $5: 1-13$

Trzesniewski KH, Donnellan MB. 2014. "Young People These Days...” Evidence for Negative Perceptions of Emerging Adults. Emerging Adulthood. 2(3):211-226.

Tustin K, Chee K-S, Taylor N, Gollop M, Taumoepeau M, Hunter J, Harold G, Poulton R. 2012. Extended baseline report: Graduate longitudinal study New Zealand. Dunedin, New Zealand.

Tustin K, Gollop M, Theodore R, Taumoepeau M, Taylor N, Hunter J, Chapple S, Chee K, Poulton R. 2016. First follow-up descriptive report: Graduate longitudinal study New Zealand. Dunedin, New Zealand.

Woolston C. 2017. Graduate survey: A love-hurt relationship. [accessed 2020 January 5]. https://www.nature.com/articles/nj7677-549a.

Woolston C. 2019. PhDs: the tortuous truth. Nature. 575:403-406. 

Table 1. Fixed effects from multilevel Bayesian regression with $95 \%$ credible intervals.

\begin{tabular}{lcccc}
\hline & \multicolumn{4}{c}{ 95\% Credible Interval } \\
\hline Intercept & Estimate & Lower & Upper & PP \\
Time (2014) & 0.01 & -0.05 & 0.03 & $67.5 \%$ \\
PhD entrant & 0.07 & 0.04 & 0.10 & $100.0 \%$ \\
Sex (female) & 0.01 & -0.11 & 0.12 & $53.6 \%$ \\
Economic strain & -0.04 & -0.09 & -0.02 & $96.5 \%$ \\
Age & 0.26 & 0.24 & 0.28 & $100.0 \%$ \\
Time (2014) * PhD entrant & 0.10 & 0.08 & 0.13 & $100.0 \%$ \\
\hline
\end{tabular}

NB: Posterior probability (PP) captures the probability of an effect deviating from zero in its given direction. The analysis uses an informed prior for the time by $\mathrm{PhD}$ entrant interaction and a weakly-informed prior for all other variables. 


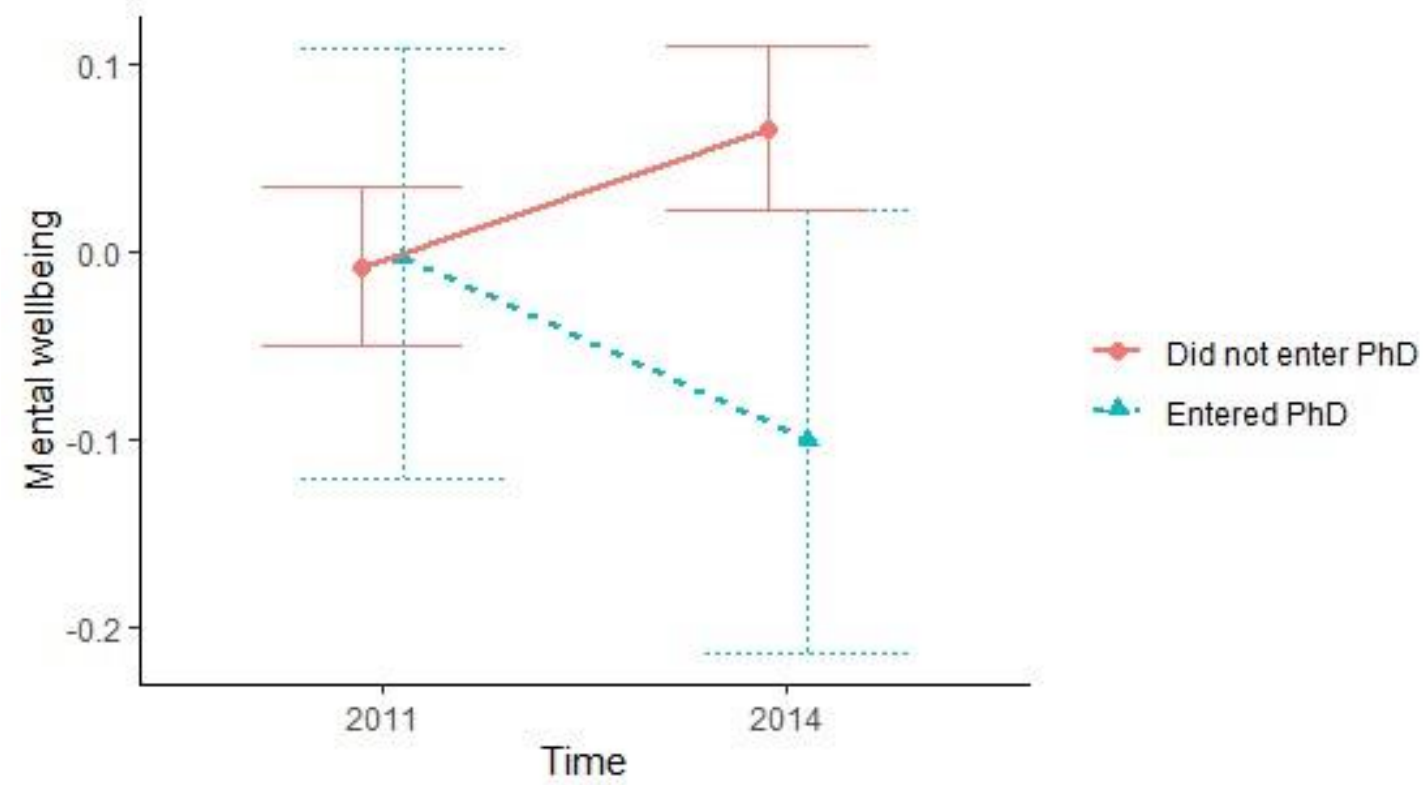

Figure 1. Marginal effects on standardised and centred mental wellbeing in 2011 and 2014 for those who did and did not transitioned into PhD study. Error bars are $95 \%$ credible intervals. 